\title{
An approximate Solution of the Laminar Boundary-layer Equation for a Flat Plate
}

\author{
By M. Yoshitake \\ Japan Meteorological Agency, Tokyo, Japan \\ (Manuscript received 3 March, 1971)
}

\begin{abstract}
The Blasius equation for a flat plate in uniform laminar flow is solved approximately in a closed form. This solution is simple but in good agreement with the exact solution found by numerical integration. Some applications of this solution are undertaken.
\end{abstract}

\section{Introduction}

The problem considered in this paper concerns the classical boundary-layer solution for a flat plate in a uniform stream. This problem is reduced to solve the Blasius equation

$$
f^{\prime \prime \prime}+f f^{\prime \prime}=0,
$$

where primes denote differentiation with respect to $\eta$; the boundary conditions being

$$
f(0)=f^{\prime}(0)=0, \quad f^{\prime}(\infty)=1 .
$$

The usual procedure is to solve the nonlinear differential equation in series expansion. Two expansions are needed for large and small $\eta$ respectively, and they are linked up. On the other hand, numerical solutions were published by Blasius (1908), Töpfer (1912), Goldstein (1930), and Howarth (1938). The numerical table for $f, f^{\prime}$, and $f^{\prime \prime}$ and some associated quantities is shown in Rosenhead (Editor) (1966) "Laminar Boundary Layers". It remains to derive the approximate solution in a closed simple form which is in good agreement with the numerical solution throughout the whole range of $\eta$.

Of course, there exist some approximate solutions in closed forms. Pohlhausen (1921) adopted a polynomial of degree one, two, three or four respectively for the velocity distribution in the boundary layer. Another form has been suggested by Lamb (1932) who takes the trigonometric form for the velocity distribution.

\section{Presumptive solution}

Refering to the numerical table of the solu- tion of the Blasius equation and bearing in mind the boundary conditions (2), let us tentatively put

$$
f^{\prime}=\sqrt{\tanh a f},
$$

where $a$ is a constant. It is needless to say that equation (3) satisfies boundary conditions $f^{\prime}(0)=0$ and $f^{\prime}(\infty)=1$.

Differentiating (3) with respect to $\eta$ and putting $f=0$, we obtain

$$
a=2 f^{\prime \prime}(0) \text {. }
$$

The constant $a$ may be determined approximately by the following procedure. Make use of the relation

$$
f^{\prime \prime}(0)=\int_{0}^{\infty} f^{\prime}\left(1-f^{\prime}\right) d \eta
$$

which can be derived from integrating by parts and taking into account (1). Inserting (3) on the right-hand side of (5)

$$
\int_{0}^{\infty} f^{\prime}\left(1-f^{\prime}\right) d \eta=\int_{0}^{\infty}(1-\sqrt{\tanh a f}) d f .
$$

To evaluate the integral we put

$$
\sqrt{\tanh a f}=x,
$$

then

$$
\begin{aligned}
& \int_{0}^{\infty}\left(1-\sqrt{\tanh a f)} d f=\frac{2}{a} \int_{0}^{1} \frac{x}{(1+x)\left(1+x^{2}\right)} d x\right. \\
& =\frac{1}{a}\left(\tan ^{-1} 1-\frac{1}{2} \log 2\right)
\end{aligned}
$$

Combining (4), (5), (6) and (7), we find. 


$$
\begin{aligned}
\frac{a}{2} & =f^{\prime \prime}(0)=\sqrt{\frac{1}{2}\left(\tan ^{-1} 1-\frac{1}{2} \log 2\right)} \\
& =0.4684 .
\end{aligned}
$$

The exact numerical value of $a / 2$ is 0.4696 . The present value is in error by 0.3 per cent.

Now we shall show that equation (3) is in good agreement with the exact solution of $f^{\prime}$. Putting

$$
a=0.9392,
$$

the approximate solution (3) is compared with the exact numerical solution. In Fig. 1 the solid

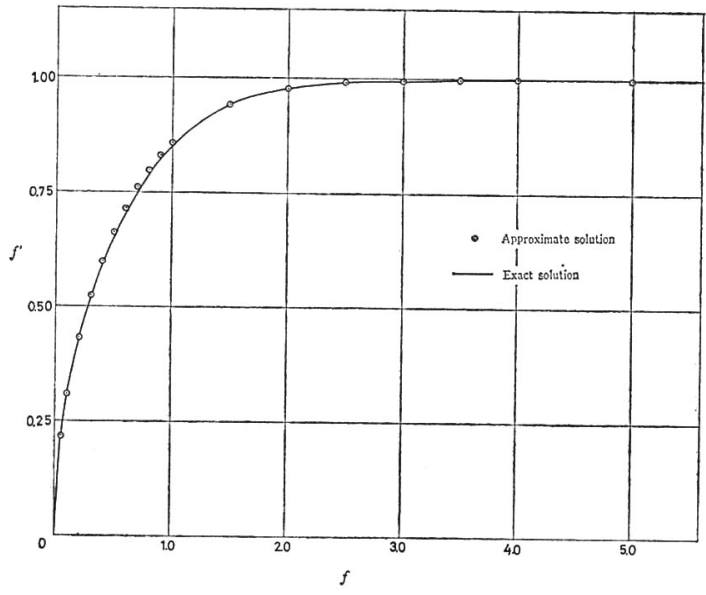

Fig. 1. Comparison between exact solution and approximate solution of $f^{\prime}$.

curve indicates the results of numerical integration shown in Rosenhead (1966) "Laminar Boundary Layers". The centers of small circles indicate the calculated values from (3) and (9), which are shown in Table 1.

We now proceed to the integration of (3). This can be done easily;

$$
\eta=\frac{1}{a}\left(\tanh ^{-1} \sqrt{\tanh a f}+\tan ^{-1} \sqrt{\tanh a f}\right),
$$

where the boundary condition $f(0)=0$ is taken into account. Equation (10) is transformed into the following form:

$$
\begin{gathered}
\eta=f+\frac{1}{a}\left\{\operatorname { l o g } \left(1+\sqrt{\tanh a f)}-\frac{1}{2} \log 2\right.\right. \\
\left.+\frac{1}{2} \log \left(1+e^{-2 a f}\right)+\tan ^{-1} \sqrt{\tanh a f}\right\} .
\end{gathered}
$$

Equation (10) or (11) gives an approximate
Table 1 Approximate solution of the Blasius equation

\begin{tabular}{c|c|c|c|c}
\hline \hline$f$ & $\eta$ & $f^{\prime}$ & $f^{\prime \prime}$ & $-f^{\prime \prime \prime}$ \\
\cline { 5 - 6 } 0.00 & 0.00000 & 0.00000 & 0.46960 & 0.00000 \\
0.05 & 0.46165 & 0.21672 & 0.46856 & 0.00896 \\
0.1 & 0.65274 & 0.30598 & 0.46548 & 0.02506 \\
0.2 & 0.92388 & 0.43084 & 0.45342 & 0.06811 \\
0.3 & 1.13335 & 0.52396 & 0.43421 & 0.11732 \\
0.4 & 1.31110 & 0.59912 & 0.40909 & 0.16525 \\
0.5 & 1.47009 & 0.66173 & 0.37955 & 0.20659 \\
& & & & \\
0.6 & 1.61501 & 0.71455 & 0.34718 & 0.23793 \\
0.7 & 1.75136 & 0.75938 & 0.31344 & 0.25782 \\
0.8 & 1.87909 & 0.79747 & 0.27967 & 0.26643 \\
0.9 & 2.00193 & 0.82982 & 0.24693 & 0.26504 \\
1.0 & 2.12038 & 0.85723 & 0.21132 & 0.25005 \\
& & & & \\
1.5 & 2.67293 & 0.94193 & 0.09993 & 0.15687 \\
2.0 & 3.19227 & 0.97689 & 0.04193 & 0.07343 \\
2.5 & 3.69494 & 0.99091 & 0.01685 & 0.03080 \\
3.0 & 4.20372 & 0.99644 & 0.00665 & 0.01236 \\
3.5 & 4.70398 & 0.99860 & 0.00262 & 0.00490 \\
4.0 & 5.20544 & 0.99946 & 0.00102 & 0.00191 \\
5.0 & 6.20124 & 0.99992 & 0.00016 & 0.00031 \\
\hline
\end{tabular}

solution of the Blasius equation. Although (10) satisfies the boundary conditions (2), but it does not satisfy the Blasius equation (1). This will be discussed in section 5. Equation (10) is a transcendental equation and we can not solve the equation analytically with respect to $f(\eta)$. Putting $a=0.9392$, the approximate solution will be compared with the exact solution. In Fig. 2 the solid curve is plotted accordingly to the table given by Rosenhead (1966). The points corresponding to the numerical values in Table 1 are found very close to the curve.

For large $f$ we have

$$
\eta \sim f+\frac{1}{a}\left(\frac{1}{2} \log 2+\tan ^{-1} 1\right)-\frac{1}{a} e^{-2 a f} .
$$

Thus we find

$$
\lim _{f \rightarrow \infty}(\eta-f)=\frac{1}{a}\left(\frac{1}{2} \log 2+\tan ^{-1} 1\right) .
$$

Putting $a=0.9392$, we have

$$
\lim _{f \rightarrow \infty}(\eta-f)=1.2052
$$




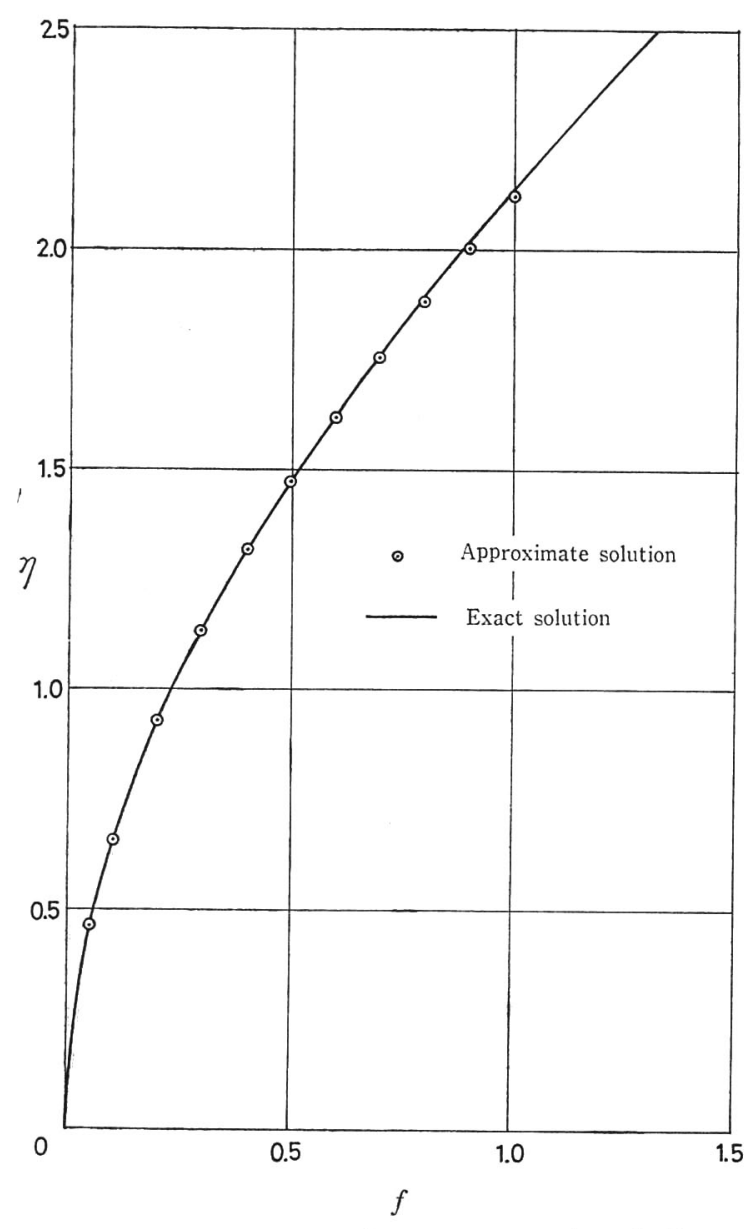

Fig. 2. Comparison between exact solution and approximate solution of $\eta(f)$.

which corresponds to the exact numerical value 1.21678. The present value is in error about one per cent.

For small $f$, equation (10) gives

$$
\eta \sim \frac{2}{a} \sqrt{\tanh a f},
$$

or inversely

$$
f \sim \frac{1}{a} \tanh ^{-1}\left(\frac{1}{2} a \eta\right)^{2} .
$$

Expanding in power series

$$
f \sim \frac{1}{4} a \eta^{2}+\frac{1}{192} a^{5} \eta^{6}+\cdots .
$$

The first term on the right-hand side just coincides with the first term of the exact solution expressed in power series, but the second term is quite different from the second term $-a^{2} \eta^{5} / 5$ ! of the exact solution. Numeri- cally it makes little difference.

We shall consider $f^{\prime \prime}$ given by differentiating (3)

$$
f^{\prime \prime}=\frac{a}{2} \operatorname{sech}^{2} a f .
$$

On the other hand, directly from the Blasius equation (1), we have

$$
f^{\prime \prime}=\frac{a}{2} \exp \left(-\int_{0}^{\eta} f d \eta\right) .
$$

In Fig. 3 the solid curve is plotted according to the table given by Rosenhead (1966). The points corresponding to the numerical values in Table 1 are found close to the curve, but the degree of agreement is not so good as in the cases $\eta$ and $f^{\prime}$. This will be discussed in section 5 .

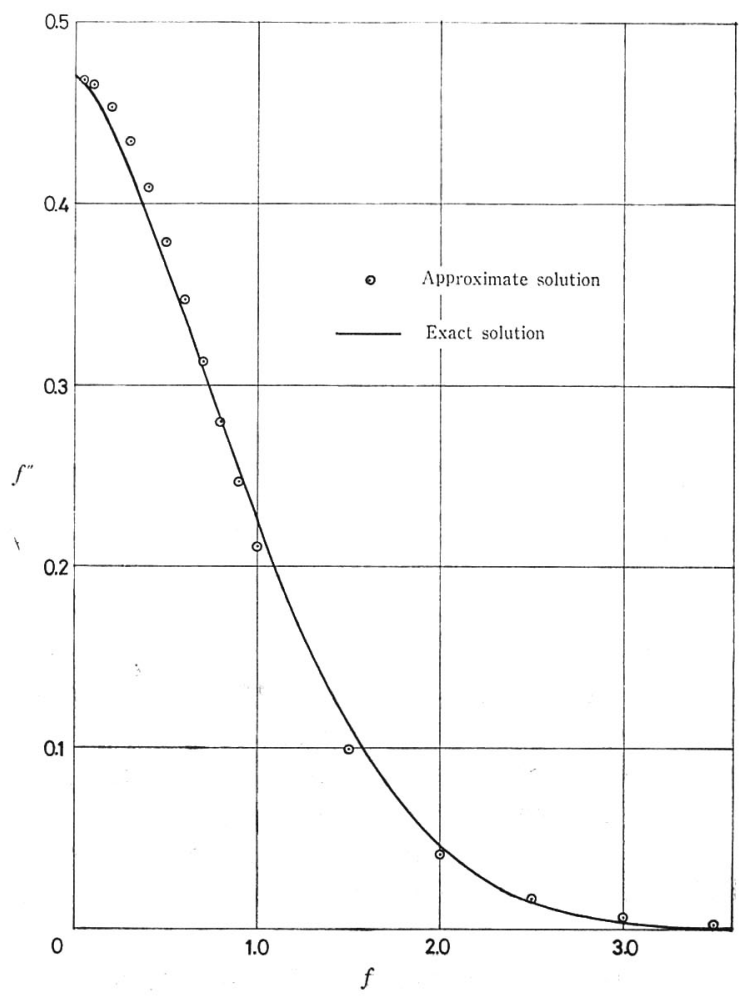

Fig. 3. Comparison between exact solution and approximate solution of $f^{\prime \prime}$.

\section{Some examples of application of equation (3)}

We shall show some examples which involve $f^{\prime}, f^{\prime \prime}$ in integrands. These example will show good agreement with the exact numerical results. 


\section{(a) Displacement thickness}

Displacement thickness $\delta_{1}$ is equal to

$$
\delta_{1}=\left(2 \nu x / U_{0}\right)^{\frac{1}{2}} \int_{0}^{\infty}\left(1-f^{\prime}\right) d \eta,
$$

where $U_{0}$ is the speed in a uniform stream outside the boundary-layer, $v$ is the coefficient of viscosity, $x$ is measured downstream along the plate. To evaluate the integral we make use of (3); whence

$$
\delta_{1}=\left(2 \nu x / U_{0}\right)^{\frac{1}{2}} \int_{0}^{\infty} \frac{1-\sqrt{\tanh a f}}{\sqrt{\tanh a f}} d f .
$$

We have now to evaluate the integral

$$
I_{1}=\int_{0}^{\infty} \frac{1-\sqrt{\tanh a f}}{\sqrt{\tanh a f}} d f .
$$

Putting $\sqrt{\tanh a f}=x$, we have

$$
\begin{aligned}
I_{1} & =\frac{2}{a} \int_{0}^{1} \frac{1}{(1+x)\left(1+x^{2}\right)} d x \\
& =\frac{1}{a}\left(\frac{1}{2} \log 2+\tan ^{-1} 1\right)=1.2052 .
\end{aligned}
$$

Thus we find

$$
\delta_{1}=1.7044\left(\nu x / U_{0}\right)^{\frac{1}{2}} \text {. }
$$

The exact numerical value of the coefficient of $\delta_{1}$ is 1.7208. The error is about one per cent.

The integral $I_{1}$ may be easily evaluated if we make use of (13), viz.

$$
I_{1}=\lim _{\eta \rightarrow \infty} \int_{0}^{\eta}\left(1-f^{\prime}\right) d \eta=\lim _{\eta \leftarrow \infty}(\eta-f)
$$

\section{(b) Momentum thickness}

The momentum thickness $\delta_{2}$ is equal to

$$
\delta_{2}=\left(2 \nu x / U_{0}\right)^{\frac{1}{2}} \int_{0}^{\infty} f^{\prime}\left(1-f^{\prime}\right) d \eta .
$$

We have already evaluated the integral in

(6) and (7). Putting $a=0.9392$ we have

$$
\begin{aligned}
& \int_{0}^{\infty} f^{\prime}\left(1-f^{\prime}\right) d \eta=\frac{1}{a}\left(\tan ^{-1} 1-\frac{1}{2} \log 2\right) \\
& =0.4672 .
\end{aligned}
$$

Thus we find

$$
\delta_{2}=0.6607\left(\nu x / U_{0}\right)^{\frac{1}{2}}
$$

The exact numerical value of the coefficient is 0.6641 . The error is about 0.5 per cent.

\section{(c) Energy thickness}

The energy thickness $\delta_{3}$ is equal to

$$
\delta_{3}=\left(2 \nu x / U_{0}\right)^{\frac{1}{2}} \int_{0}^{\infty} f^{\prime}\left(1-f^{\prime 2}\right) d \eta .
$$

Put

$$
I_{3}=\int_{0}^{\eta} f^{\prime}\left(1-f^{\prime 2}\right) d \eta
$$

Making use of (3)

$$
\begin{aligned}
I_{3} & =\int_{0}^{f}(1-\tanh a f) d f=f-\frac{1}{a} \log \cosh a f \\
& =-\frac{1}{a} \log \left(1+e^{-2 a f}\right)+\frac{1}{a} \log 2 .
\end{aligned}
$$

Thus we have

$$
\int_{0}^{\infty} f^{\prime}\left(1-f^{\prime 2}\right) d \eta=\frac{1}{a} \log 2=0.73802 .
$$

The exact numerical value of this integral is 0.73849 . The error is about 0.07 per cent. Finally we find

$$
\delta_{3}=1.0437\left(\nu x / U_{0}\right)^{\frac{1}{2}}
$$

\section{(d) The integral}

$$
\int_{0}^{\infty} f^{\prime \prime}(\eta) f^{\prime}(\eta) \eta d \eta
$$

This definite integral was evaluated by Meksyn (1956) by the method of steepest descent. We shall now evaluate the integral by making use of (3).

Integrating by parts we have

$$
\int_{0}^{\eta} f^{\prime \prime} f^{\prime} \eta d \eta=\frac{1}{2} f^{\prime 2} \eta-\frac{1}{2} \int_{0}^{\eta} f^{\prime 2} d \eta
$$

Make use of (3)

$$
\begin{aligned}
& \int_{0}^{\eta} f^{\prime \prime} f^{\prime} \eta d \eta=\frac{1}{2} \eta \tanh a f- \\
& \frac{1}{2 a}\left(\tanh ^{-1} \sqrt{\tanh a f}-\tan ^{-1} \sqrt{\tanh a f}\right) .
\end{aligned}
$$

Then

$$
\begin{aligned}
& \int_{0}^{\infty} f^{\prime \prime} f^{\prime} \eta d \eta=\frac{1}{2 a}\left(\tan ^{-1} 1-\frac{1}{2} \log 2\right) \\
& +\frac{1}{2} \lim _{\eta \rightarrow \infty}(\eta-f)
\end{aligned}
$$

Combining (13) and (36) 


$$
\int_{0}^{\infty} f^{\prime \prime} f^{\prime} \eta d \eta=\frac{1}{a} \tan ^{-1} 1=0.8362
$$

This numerical value stands against 0.8414 given by Meksyn. The error is about 0.6 per cent.

\section{Semi-infinite plane at constant tempera- ture}

This problem will be considered in detail by making use of the approximate solution of the Blasius equation.

The equation of motion, continuity and energy are

$$
\begin{aligned}
& u \frac{\partial u}{\partial x}+v \frac{\partial u}{\partial y}=-\frac{1}{\rho} \frac{\partial p}{\partial x}+\nu \frac{\partial^{2} u}{\partial y^{2}}, \\
& \frac{\partial u}{\partial x}+\frac{\partial v}{\partial y}=0, \\
& \rho c_{p}\left(u \frac{\partial T}{\partial x}+v \frac{\partial T}{\partial y}\right)=k \frac{\partial^{2} T}{\partial y^{2}}+u \frac{\partial T}{\partial x} \\
& +\mu\left(\frac{\partial u}{\partial y}\right)^{2} .
\end{aligned}
$$

In the present case $\partial p / \partial x=0$.

Changing the variable, let

$$
\eta=\left(U_{0} / 2 \nu x\right)^{\frac{1}{2}} y, \quad \phi=\left(2 \nu U_{0} x\right)^{\frac{1}{2}} f(\eta),
$$

where $U_{0}$ is the velocity at infinity, we find

$$
\begin{aligned}
& u=\frac{\partial \psi}{\partial y}=U_{0} f^{\prime}, \\
& v=-\frac{\partial \psi}{\partial x}=\left(\nu U_{0} / 2 x\right)^{\frac{1}{2}}\left[\eta f^{\prime}-f(\eta)\right], \\
& \frac{d^{2} T}{d \eta^{2}}+\sigma f \frac{d T}{d \eta}+\frac{\mu U_{0}^{2}}{k} f^{\prime \prime 2}=0,
\end{aligned}
$$

where $\sigma=\mu c_{p} / k$ is the Prandtl number.

The equation of motion is

$$
f^{\prime \prime \prime}+f f^{\prime \prime}=0,
$$

with the usual boundary condition

$$
f(0)=f^{\prime}(0)=0, \quad f^{\prime}(\infty)=1 .
$$

The boundary conditions for temperature are

$$
T=T_{w} \text { at } \eta=0, \quad T=T_{\infty} \text { at } \eta=\infty .
$$

The solution of (40) which satisfies the boundary condition at $\eta=0$ is

$$
T=T_{w}+\left(\frac{d T}{d \eta}\right) \int_{0}^{\eta} e^{-\sigma F} d \eta
$$

$$
-\frac{\mu U_{0}^{2}}{k} \int_{0}^{\eta} e^{-\sigma F} d \eta-\int_{0}^{\eta} e^{\sigma F} f^{\prime \prime 2} d \eta
$$

where

$$
F=\int_{0}^{\eta} f(\eta) d \eta
$$

The temperature gradient at $\eta=0$ is found from the boundary condition at infinity, i.e.

$$
\begin{aligned}
& T_{\infty}=T_{w}+\left(\frac{d T}{d \eta}\right) \int_{0}^{\infty} e_{0}^{-\sigma F} d \eta-\frac{\mu U_{0}^{2}}{k} \\
& \times \int_{0}^{\infty} e^{-\sigma F} d \eta \int_{0}^{\eta} e^{\sigma F} f^{\prime \prime 2} d \eta .
\end{aligned}
$$

First we disregard in (46) the term due to dissipation of energy. From (18), (19) and (45) we have

$$
e^{-F}=\operatorname{sech}^{2} a f,
$$

whence we have

$$
T_{\infty}=T_{w}+\left(\frac{d T}{d \eta}\right) \int_{0}^{\infty} \operatorname{sech}^{2^{\sigma}} a f \cdot \frac{d \eta}{d f} \cdot d f .
$$

Put

$$
\tanh a f=\sqrt{x},
$$

then the integral on the right-hand side of (48) becomes

$$
\int_{0}^{\infty} \frac{\operatorname{sech}^{2 \sigma} a f}{\sqrt{\tanh a f}} d f=\frac{1}{2 a} \int_{0}^{1} x^{\frac{1}{4}-1}(1-x)^{\sigma-1} d x .
$$

Integrating in the Beta function we find

$$
T_{\infty}=T_{w}+\left(\frac{d T}{d \eta}\right)_{0} \frac{1}{2 a} B\left(\frac{1}{4}, \sigma\right) .
$$

Solving (50) with respect to $(d T / d \eta)_{0}$,

$$
\left(\frac{d T}{d \eta}\right)_{0}=2 a \frac{\Gamma\left(\frac{1}{4}+\sigma\right)}{\Gamma\left(\frac{1}{4}\right) \Gamma(\sigma)}\left(T_{\infty}-T_{w}\right),
$$

where use is made of the relation between the Beta and Gamma functions. For the case $\sigma=0.72$ we find

$$
\left(\frac{d T}{d \eta}\right)_{0}=0.416\left(T_{\infty}-T_{w}\right)
$$

to be compared with the numerical value 0.415 given by Meksyn (1961).

Equation (51) is used for small $\sigma$. For large $\sigma$ there appears considerable error. 
We put

$$
C=2 a \frac{\Gamma\left(\frac{1}{4}+\sigma\right)}{\Gamma\left(\frac{1}{4}\right) \Gamma(\sigma)} .
$$

In Table 2 are shown the numerical values of $C$ calculated from (53). In the same table are inserted the numerical values of $C$ evaluated by Pohlhausen (1921). We see good agreement for the range $\sigma=0.6 \sim 1.1$. Figure 4 shows the variation of $C$ with $\sigma$ in logarithmic scale. The straight dotted line is drawn in accord with $C=0.468 \sigma^{1 / 3}$ suggested by Polhausen (1921).

Table 2 Variation $C$ with $\sigma$.

\begin{tabular}{c|c|c|c|c}
\hline \hline$\sigma$ & $C$ & & $\sigma$ & $C$ \\
\hline 0.1 & 0.139 & & 1.6 & 0.548 \\
0.2 & 0.222 & & 1.7 & 0.559 \\
0.3 & 0.280 & & 1.8 & 0.569 \\
0.4 & 0.322 & (Pohl- & 1.9 & 0.578 \\
0.5 & 0.358 & hausen $)$ & 2.0 & 0.587 \\
& & & & \\
0.6 & 0.390 & 0.390 & 2.1 & 0.596 \\
0.7 & 0.412 & 0.413 & 2.2 & 0.604 \\
0.8 & 0.434 & 0.434 & 2.3 & 0.612 \\
0.9 & 0.452 & 0.453 & 2.4 & 0.619 \\
1.0 & 0.470 & 0.470 & 2.5 & 0.627 \\
& & & & \\
1.1 & 0.485 & 0.486 & & \\
1.2 & 0.500 & & & \\
1.3 & 0.513 & & & \\
1.4 & 0.526 & & & \\
1.5 & 0.537 & & & \\
\hline
\end{tabular}

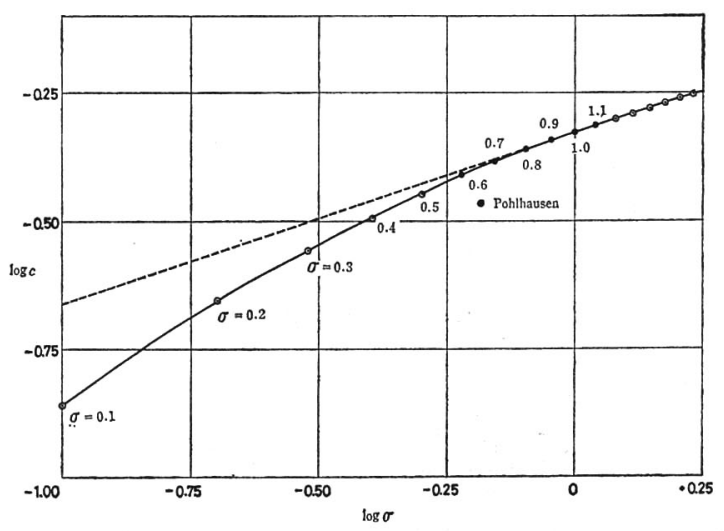

Fig. 4. $(\log C)$ calculated for diffeernt values of $\sigma$.
Now we return to equation (44). Making use of (47), (44) becomes

$$
\begin{aligned}
& T=T_{w}+\left(\frac{d T}{d \eta}\right) \int_{0}^{f} \frac{\operatorname{sech}^{2 \sigma} a f}{\sqrt{\tanh a f}} d f-\frac{\mu U_{0}^{2}}{k} \frac{a^{2}}{4} \\
& \times \int_{0}^{f} \frac{\operatorname{sech}^{2 \sigma} a f}{\sqrt{\tanh a f}} d f \int_{0}^{f} \frac{\operatorname{sech}^{2(2-\sigma)} a f}{\sqrt{\tanh a f}} d f .
\end{aligned}
$$

Changing the independent variable, let

$$
f=\frac{1}{a} \tanh ^{-1} x^{2},
$$

whence

$$
\begin{aligned}
& T=T_{w}+\left(\frac{d T}{d \eta}\right)_{0} \frac{2}{a} \int_{0}^{x}\left(1-x^{4}\right)^{\sigma-1} d x-\frac{\mu U_{0}^{2}}{k} \\
& \times \int_{0}^{x}\left(1-x^{4}\right)^{\sigma-1} d x \int_{0}^{x}\left(1-x^{4}\right)^{-(\sigma-1)} d x .
\end{aligned}
$$

For the case $\sigma=1$

$$
\begin{aligned}
& T=T_{w}+\left(\frac{d T}{d \eta}\right)_{0} \frac{2}{a} \sqrt{\tanh a f}-\frac{\mu U_{0}^{2}}{k} \\
& \times \frac{1}{2} \tanh a f .
\end{aligned}
$$

Put $f=\infty$, then

$$
T_{\infty}=T_{w}+\left(\frac{d T}{d \eta}\right)_{0} \frac{2}{a}-\frac{\mu U_{0}^{2}}{k} \cdot \frac{1}{2} .
$$

For the case $\sigma=2$ we have

$$
\begin{aligned}
& T=T_{w}+\left(\frac{d T}{d \eta}\right)_{0} \frac{2 x}{a}\left(1-\frac{1}{5} x^{4}\right) \\
& -\frac{\mu U_{0}^{2}}{k}\left\{\frac{1}{2} x\left(1-\frac{1}{5} x^{4}\right)\left(\tanh ^{-1} x+\tan ^{-1} x\right)\right. \\
& \left.-\frac{2}{5} \tanh ^{-1} x^{2}-\frac{1}{10} x^{2}\right\}
\end{aligned}
$$

Turning back to the variable $f$

$$
\begin{aligned}
& T=T_{w}+\left(\frac{d T}{d \eta}\right)_{0} \frac{2}{a} \sqrt{\tanh a f}\left(1-\frac{1}{5} \tanh ^{2} a f\right) \\
& -\frac{\mu U_{0}^{2}}{k}\left\{\frac{1}{2} \sqrt{\tanh a f}\left(1-\frac{1}{5} \tanh ^{2} a f\right) a \eta(f)\right. \\
& \left.-\frac{2}{5} a f-\frac{1}{10} \tanh a f\right\}
\end{aligned}
$$

where

$\eta(f)=\frac{1}{a}\left(\tanh ^{-1} \sqrt{\tanh a f}+\tan ^{-1} \sqrt{\tanh a f}\right)$. 
This is the analytical expression of temperature profile for the case $\sigma=2$. Putting $f=\infty$, we have

$$
\begin{aligned}
& T_{\infty}=T_{w}+\left(\frac{d T}{d \eta}\right)_{0} \frac{8}{5 a}-\frac{\mu U_{0}{ }^{2}}{k} \\
& \times\left\{\frac{2 a}{5} \lim _{f \rightarrow \infty}(\eta-f)-\frac{1}{10}\right\} .
\end{aligned}
$$

From (13) and (62)

$$
\begin{aligned}
& \left(\frac{d T}{d \eta}\right)_{0}=\frac{5 a}{8}\left[T_{\infty}-T_{w}+\frac{\mu U_{0}^{2}}{k}\right. \\
& \left.\times\left\{\frac{2}{5}\left(\frac{1}{2} \log 2+\tan ^{-1} 1\right)-\frac{1}{10}\right\}\right] .
\end{aligned}
$$

We shall now consider the problem of a plate thermometer. This problem is to find the equilibrium temperature of the plate. The temperature of the plate $T_{w}$ is given by putting $(d T / d \eta)_{0}=0$ and $x \rightarrow 1$ in (56), i.e.

$$
\begin{aligned}
& T_{w}=T_{\infty}+\frac{\mu U_{0}^{2}}{k} \\
& \times \lim _{x \rightarrow 1} \int_{0}^{x}\left(1-x^{4}\right)^{\sigma-1} d x \int_{0}^{x}\left(1-x^{4}\right)^{-(\sigma-1)} d x .
\end{aligned}
$$

Generally speaking the consecutive integral in (64) can be evaluated analytically when $\sigma$ takes integral numbers. In the process of integration we make use of the following recurrence formula:

$$
\begin{aligned}
& \int \frac{d x}{\left(1-x^{4}\right)^{\sigma}}=\frac{1}{4(\sigma-1)} \frac{x}{\left(1-x^{4}\right)^{\sigma-1}} \\
& +\frac{4 \sigma-5}{4 \sigma-4} \int \frac{d x}{\left(1-x^{4}\right)^{\sigma-1}} .
\end{aligned}
$$

Put

$$
T_{w}=T_{\infty}+\frac{U_{0}^{2}}{2 c_{p}} \theta_{\sigma}(0),
$$

then

$$
\begin{aligned}
& \theta_{\sigma}(0)=2 \sigma \\
& \times \lim _{x \rightarrow 1} \int_{0}^{x}\left(1-x^{4}\right)^{\sigma-1} d x \int_{0}^{x}\left(1-x^{4}\right)^{-(\sigma-1)} d x .
\end{aligned}
$$

After somewhat laborious mathematical calculations, we have the following results:

$$
\begin{aligned}
& \theta_{1}(0)=1 \\
& \theta_{2}(0)=\frac{8}{5}\left(\frac{1}{2} \log 2+\tan ^{-1} 1\right)-\frac{\varepsilon}{5}=1.411 \\
& \theta_{3}(0)=\frac{8}{5}\left(\frac{1}{2} \log 2+\tan ^{-1} 1\right)-\frac{1}{15}=1.756 \\
& \theta_{4}(0)=\frac{112}{65}\left(\frac{1}{2} \log 2+\tan ^{-1} 1\right)+\frac{16}{195}=2.045 \\
& \theta_{5}(0)=\frac{1232}{663}\left(\frac{1}{2} \log 2+\tan ^{-1} 1\right)+\frac{138719}{891072}=2.274
\end{aligned}
$$

In Fig. 5 are plotted the numerical values of $\theta_{\sigma}(0)$ for $\sigma=1,2,3,4$ and 5. There are also plotted the results by Pohlhausen (1921) for

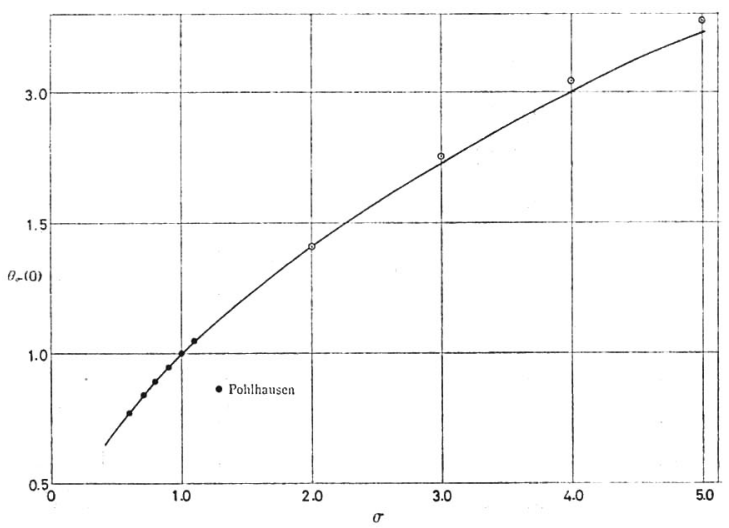

Fig. 5. $\theta_{\sigma}(0)$ calculated for different values of $\sigma$.

$\sigma=0.6,0.7,0.8,0.9,1.0$ and 1.1. In the same figure is drawn a curve corresponding to the approximate form $\theta_{\sigma}(0)=\sigma^{1 / 2}$ suggested by Pohlhausen. The calculated values for $\sigma=3,4$ and 5 lie slightly above this curve.

\section{Approximate solution}

In this section we shall consider the approximate solution $f^{\prime}=\sqrt{\tanh a f}$ itself. To make clear distinction between the approximate function and the exact function, we add subscript 1 for the approximate function:

$$
f_{1}^{\prime}=\sqrt{\tanh a f},
$$

then we have

$$
f_{1}^{\prime \prime}=\frac{a}{2} \operatorname{sech}^{2} a f=\frac{a}{2}\left(1-f_{1}{ }^{4}\right) .
$$

Further differentiation leads to

$$
f_{1}^{\prime \prime \prime}=-2 a f_{1}{ }^{3} f_{1}^{\prime \prime} \text {. }
$$




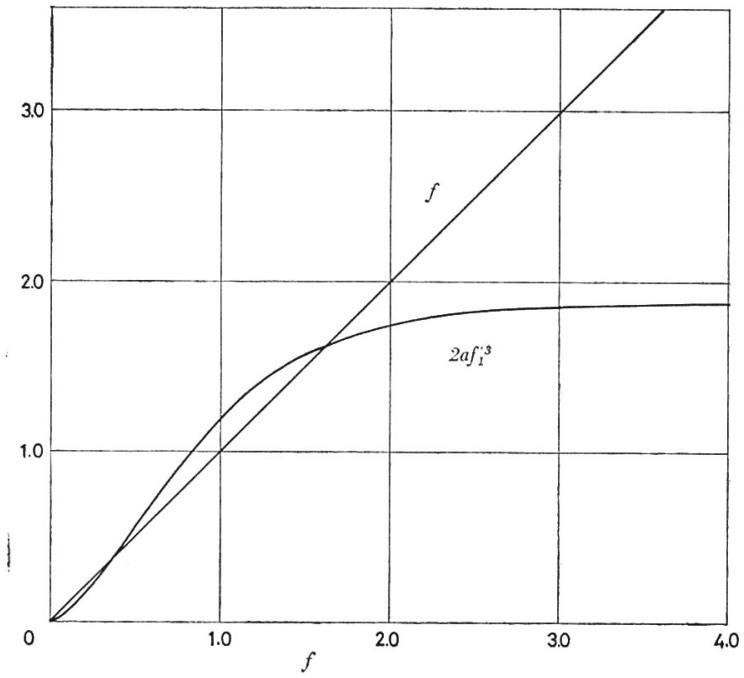

Fig. 6. Comparison between $f$ and $2 a f_{1}{ }^{3}$.

Comparing this equation with the Blasius equation (1), we know that we have replaced $f$ by $2 a f^{\prime 3}$. In Fig. 6 are shown the curve $2 a f_{1}{ }^{\prime 3}$ and the straight line $f$. Within the range $f=0 \sim 2$, the agreement is reasonably good, though not as good as that between $f_{1}^{\prime \prime}$ and $f^{\prime \prime}$. For $f>2$, they begin to separate each other. It may be said that we made a daring approximation. Fig. 7 shows the relation between

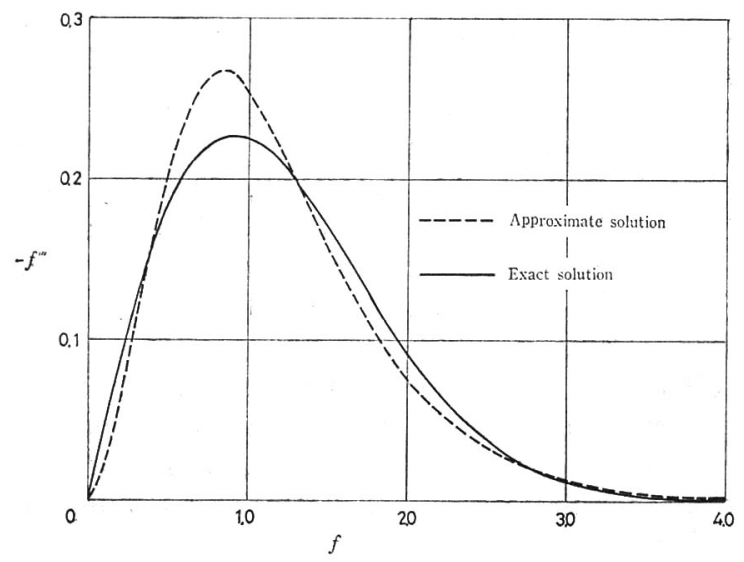

Fig. 7. Comparison between exact solution and approximate solution of $f^{\prime \prime \prime}$. the approximate solution $f_{1}^{\prime \prime \prime}$ and the exact solution $f^{\prime \prime \prime}$. Here we see reasonably good agreement throughout the whole range of $f$. The functions $f_{1}^{\prime \prime}$ and $f^{\prime \prime}$ diminish exponentially for large $f$. Fortunately this diminishing prevents the separation between $2 \alpha f_{1}{ }^{\prime 3} f_{1}{ }^{\prime \prime}$ and $f f^{\prime \prime}$ for large $f$. The degrees of agreement are improved with the advance of integration as shown in Fig. 3 and Fig. 1.

\section{Conclusion}

It was shown that the approximate solution shows good agreement with the exact solution and that it is useful for problems relating to a semi-infinite plate in uniform laminar flow. Generally speaking, it is a very difficult problem to find such a solution of another nonlinear differential equation which appears in the boundary-layer theory. However, I expect that an example which is shown in this short note will afford a key to solution of the problem.

\section{References}

Blasius, H., (1908): Grenzschichten in Flüssigkeiten mit kleiner Reibung. Z. Math. Phys. $\mathbf{5 6}, 1-37$.

Goldstein, S., (1930): Concerning some solutions of the boundary-layer equations in hydrodynamics. Proc. Camb. Phil. Soc. 26, 1-30.

Howarth, L., (1938): On the solution of the laminar boundary layer equations. Proc. Roy Soc. A164, 547-79.

Lamb, H., (1932) : Hydrodynamics, 6th edition. Cambridge University Press. p. 686.

Meksyn, D., (1961): New methods in laminar boundary-layer theory. Pergamon Press, Oxford.

Pohlhausen, K., (1921): Zur näherungsweisen Integration der Differentialgleichung der laminaren Grenzschicht. Zeitscher. f. angew. Math. u. Mech. 1, 115-120, 257-261.

Rosenhead, L. (Editor), (1966) : Laminar Boundary Layers, Clarendon Press, Oxford.

Töpfer, K., (1912): Bemerkung zu dem Aufsatz von H. Blasius 'Grenzschichten in Flüssigkeiten mit kleiner Reibung'. Z. Math. Phys. 60, 397-8. 